\title{
A Widely Applicable Dual-Catalytic System for Cross-Electrophile Coupling
}

David J. Charboneau, ${ }^{a}$ Emily L. Barth, ${ }^{a}$ Nilay Hazari ${ }^{\mathrm{a}, *}$ Mycah R. Uehling ${ }^{\mathrm{b}} \&$ Susan L. Zultanski ${ }^{\mathrm{c}, *}$

${ }^{a}$ Department of Chemistry, Yale University, P. O. Box 208107, New Haven, Connecticut, 06520, USA. E-mail: nilay.hazari@ yale.edu.

${ }^{b}$ Merck \& Co., Inc., Discovery Chemistry, HTE and Lead Discovery Capabilities, Kenilworth, New Jersey, 07033, USA.

'Merck \& Co., Inc., Department of Process Research and Development, Rahway, New Jersey, 07065, USA. E-mail: susan_zultanski@merck.com.

\begin{abstract}
A new dual catalytic system for cross-electrophile coupling reactions between aryl and alkyl halides that features a Ni catalyst, a Co co-catalyst, and a mild homogeneous reductant, is described. This is a unique combination of reagents for cross-electrophile coupling reactions, which results in one of the most versatile systems reported to date. For example, the coupling of aryl bromides and aryl iodides with alkyl bromides, alkyl iodides, alkyl mesylates, and benzyl chlorides is demonstrated under similar reaction conditions. The system is tolerant of numerous functional groups and is capable of coupling heteroaryl halides, di-ortho-substituted aryl halides, pharmaceutically relevant drug-like aryl halides, and a diverse range of alkyl halides. Additionally, the dual catalytic platform facilitates a series of novel one-pot three-component cross-electrophile coupling reactions of bromo(iodo)arenes with two distinct alkyl halides. Mechanistic studies indicate that the Ni catalyst activates the aryl halide electrophile, while the Co catalyst activates the alkyl electrophile.
\end{abstract}




\section{Introduction}

$\mathrm{Ni}$-catalyzed cross-electrophile coupling (CEC) reactions to generate new $\mathrm{C}\left(\mathrm{sp}^{2}\right)-\mathrm{C}\left(\mathrm{sp}^{3}\right)$ bonds have received significant attention over the last decade due to the prevalence of these linkages in natural products and pharmaceuticals, and the limitations of current synthetic methods to form these bonds (Figure 1a). ${ }^{[1]}$ Despite the widespread interest, reaction development has relied heavily on the empirical screening of reaction parameters, such as the identity of the ancillary ligand and presence of pyridine and alkali halide additives. ${ }^{[2]}$ Consequently, reaction conditions developed using this strategy are often specific to a limited range of substrates. ${ }^{[3]}$ For example, typical reaction conditions for the coupling of aryl halides with primary alkyl bromides, primary alkyl iodides, or primary benzyl chlorides utilize different ligand sets, additives, solvents, and reductants. $^{[1 \mathrm{a}, 1 \mathrm{~b}, 2,4]}$ Further, even within a particular substrate class, for instance nitrogencontaining heteroaryl halides, significant changes are often required depending on the exact identity of the substrates. ${ }^{[5]}$ Unfortunately, it is often unclear why a given alteration of reaction conditions or the presence of an additive results in the desired reactivity, which complicates the translation of the method to other substrates. These challenges have limited the application of CEC in synthetic chemistry, especially for the functionalization of complex pharmaceutically relevant substrates. ${ }^{[6]}$

A potential reason for the lack of generalizable conditions for CEC is related to the proposed mechanism, ${ }^{[7]}$ in which it is difficult to independently control key on- and off-cycle reactions (Figure 1b). Specifically, in CEC reactions between aryl and alkyl halides, complexes of the type $\mathrm{LNi}^{\mathrm{II}}(\mathrm{Ar}) \mathrm{X}(\mathrm{X}=\mathrm{Cl}, \mathrm{Br}$, or I) are proposed to be crucial intermediates, as they are both the catalyst resting state and responsible for capturing free alkyl radicals (Figure 1b). ${ }^{\text {[a] }}$ In an ideal CEC reaction, the $L N i^{I I}(A r) X$ intermediate would be stable and the rate at which radicals are generated would be controlled relative to the concentration of $L N i^{I I}(A r) X$ to facilitate effective radical capture (Figure 2). However, the rate of alkyl radical generation by a $\mathrm{Ni}^{\mathrm{I}}$ halide intermediate cannot be tuned separately from the concentration of $\mathrm{LNi}^{\mathrm{II}}(\mathrm{Ar}) \mathrm{X}$ because both complexes are intermediates on the same catalytic cycle. Additionally, under the reaction conditions typically utilized, $\mathrm{LNi}^{\mathrm{II}}(\mathrm{Ar}) \mathrm{X}$ complexes are unstable and can undergo two deleterious side reactions: (i) bimolecular decomposition to produce biaryl, ${ }^{[7 \mathrm{~d}, 8]}$ which is especially problematic when high catalyst loadings are employed, or (ii) direct reduction to produce 
a) Conventional Cross-Electrophile Coupling Conditions

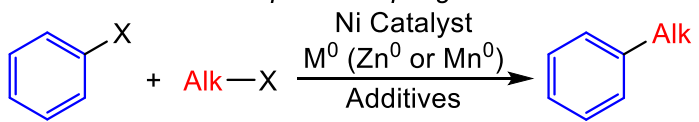

b) Proposed Mechanism

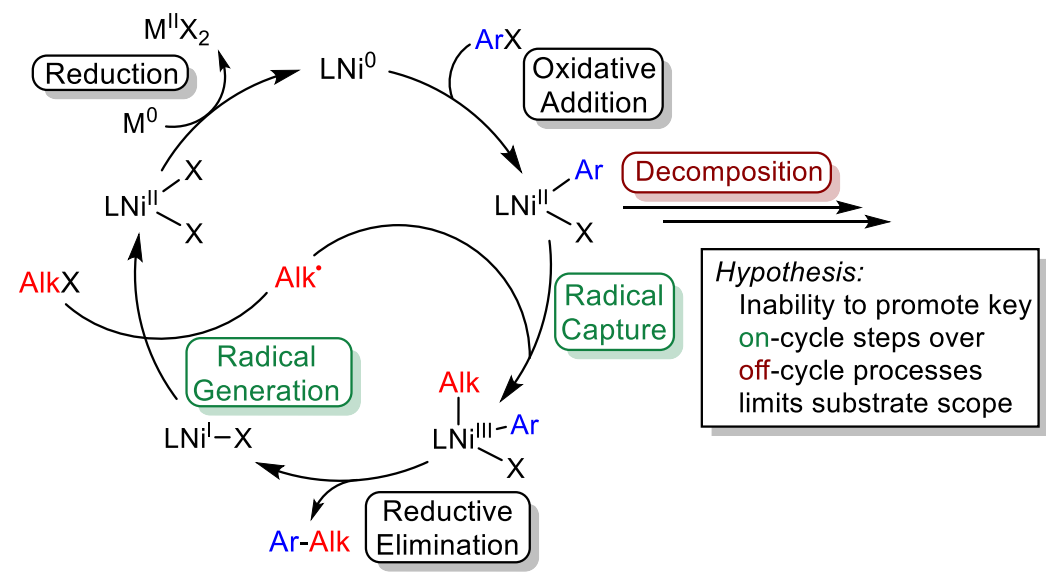

c) This Work: Novel and Widely Applicable System
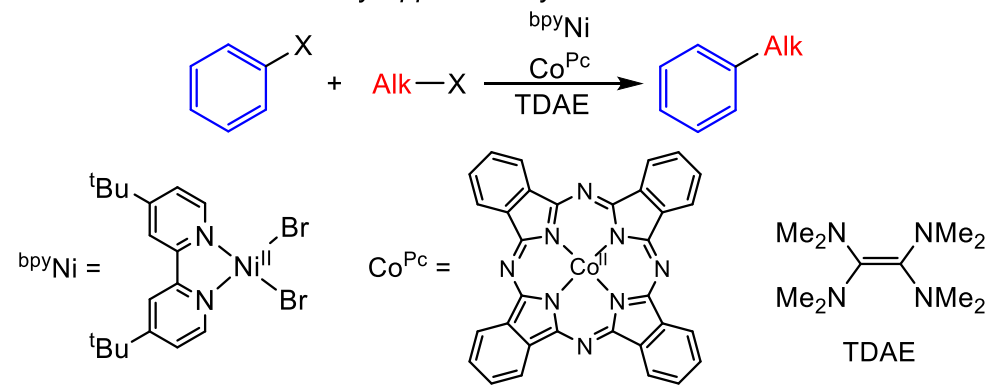

Figure 1. a) General depiction and b) mechanism of conventional Ni-catalyzed CEC reactions. $\left.{ }^{[7]} \mathbf{c}\right)$ CEC reaction described in this work.

protodehalogenated and biaryl products, which can readily occur when using strong heterogeneous $\mathrm{Zn}^{0}$ or $\mathrm{Mn}^{0}$ reductants. ${ }^{[7 \mathrm{c}, 7 \mathrm{~d}, 9]}$ Further, heterogeneous reductants present practical limitations, including challenges with process scale reactions, flow chemistry, and automated chemical synthesis, ${ }^{[10]}$ and typically require the use of toxic amide-based solvents and additives ${ }^{[4 c, 9 b, 11]}$ for efficient electron transfer to solution state catalysts. ${ }^{[10 \mathrm{~d}, 12]}$ We hypothesized that by addressing the challenges related to the stability of the $\mathrm{LNi}^{\mathrm{II}}(\mathrm{Ar}) \mathrm{X}$ intermediate and the inability to discretely control radical generation we could develop a general and practical system for $\mathrm{C}\left(\mathrm{sp}^{2}\right)-\mathrm{C}\left(\mathrm{sp}^{3}\right) \mathrm{CEC}$ (Figure 2).

Here, we report an operationally simple protocol for CEC reactions between aryl and alkyl halides by using a Ni catalyst, a radical generating Co co-catalyst and a weak homogeneous reductant (Figure 1c). The Co co-catalyst activates the alkyl halide, which allows for control of 


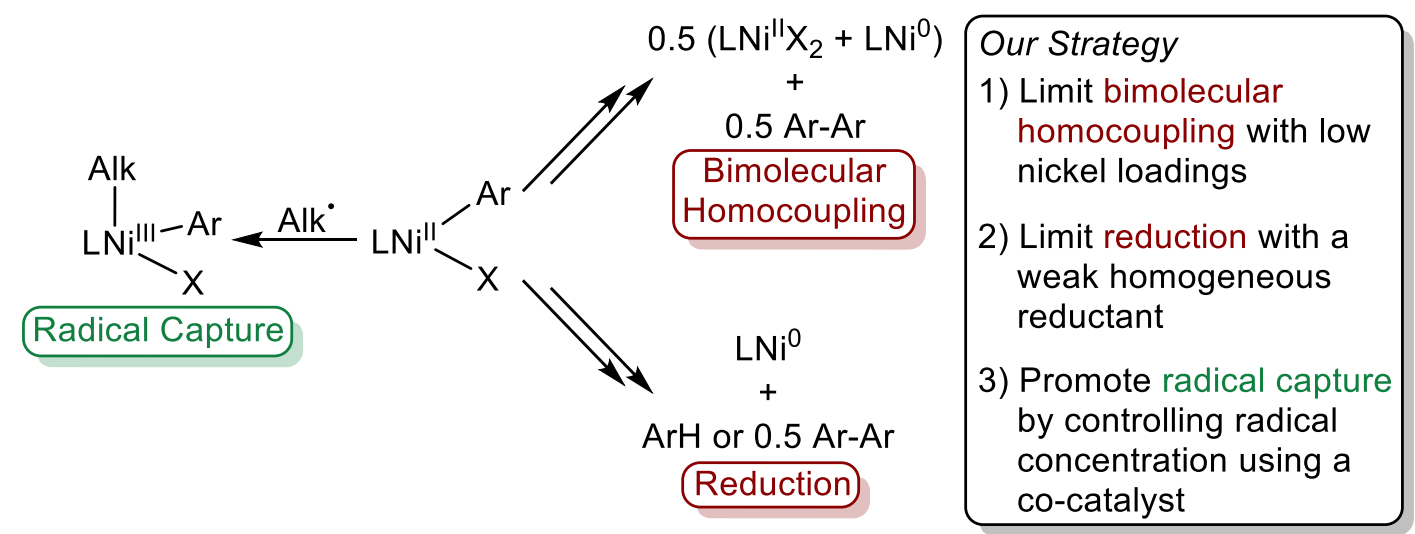

Figure 2. Potential reactions of $\mathrm{LNil}^{\mathrm{Il}}(\mathrm{Ar}) \mathrm{X}$ in catalysis and our strategy for system development.

the concentration of the alkyl radical relative to the concentration of the $\mathrm{LNi}^{\mathrm{II}}(\mathrm{Ar}) \mathrm{X}$ intermediate (vide infra). The weak homogeneous reductant and generally low Ni loadings limit decomposition of $\mathrm{LNi}^{\mathrm{II}}(\mathrm{Ar}) \mathrm{X}$. The reactions do not require additives and are easy to optimize because the product profile contains information about how to vary the ratio of the catalysts to promote radical capture at $\mathrm{LNi}^{\mathrm{II}}(\mathrm{Ar}) \mathrm{X}$ before its decomposition in catalysis. As a result, a wide range of aryl and alkyl halides can be coupled in high yield, including substrates that are rarely utilized in CEC, such as di-ortho-substituted arenes and a diverse array of medicinally relevant substrates. Further, we show that our general strategy can facilitate the discovery of novel reactions by performing a series of one-pot three-component dialkylations of bromo(iodo)arenes with two distinct alkyl electrophiles. Finally, owing to the wide range of transformations that propose radical capture at intermediates of the type $\mathrm{LNi}^{\mathrm{II}}(\mathrm{Ar}) \mathrm{X},{ }^{[13]}$ our general strategy of controlling key processes associated with this complex may be relevant to both improving a range of current reactions and developing new methods.

\section{Results and Discussion}

Method for Reaction Optimization

We sought to identify appropriate reagents to explore our strategy of inhibiting off-cycle reactivity at $\mathrm{LNi}^{\mathrm{II}}(\mathrm{Ar}) \mathrm{X}$ intermediates by using a weak homogeneous reductant and promoting productive radical capture through the use of a co-catalyst capable of generating a radical from an alkyl halide. An established method for activating an alkyl electrophile to generate an alkyl radical under reductive conditions is to use a Co catalyst. ${ }^{[14]}$ Based on its previous compatibility in CEC with heterogeneous $\mathrm{Zn}^{0}$ or $\mathrm{Mn}^{0}$ reductants, we selected $\mathrm{Co}^{\mathrm{II}}(\mathrm{Pc})\left(\mathrm{Co}^{\mathrm{Pc}} ; \mathrm{Pc}=\right.$ phthalocyanine $) .{ }^{[15]}$ Next, we selected the weak homogeneous reductant tetrakis(dimethylamino)ethylene (TDAE), 
which is known to be compatible with Ni-catalyzed reductive coupling reactions. ${ }^{[4 c, 16]}$ Importantly, $\operatorname{TDAE}\left(\mathrm{E}^{\circ}=-0.57 \mathrm{~V}\right.$ vs NHE$)$ is a weaker reductant than $\mathrm{Mn}^{0}\left(\mathrm{E}^{\circ}=-1.19 \mathrm{~V}\right.$ vs NHE$)$ or $\mathrm{Zn}^{0}\left(\mathrm{E}^{\circ}\right.$ $=-0.76 \mathrm{~V}$ vs NHE),${ }^{\left[{ }^{6}\right]}$ which should limit deleterious reduction of $\mathrm{LNi}^{\mathrm{II}}(\mathrm{Ar}) \mathrm{X}$ species (Figure $2 \mathrm{a}$ ), but it is still capable of reducing $\mathrm{Co}^{\mathrm{Pc}}$ (vide infra) and the commonly utilized $\mathrm{Ni}$ catalyst (dtbbpy) $\mathrm{Ni}^{\mathrm{II}} \mathrm{Br}_{2}\left({ }^{\text {bpy }} \mathrm{Ni}\right.$; dtbbpy $=4,4$ '-di-tert-butyl-2,2'-bipyridine). ${ }^{[7 \mathrm{~d}]}$

To test our strategy, we performed CEC reactions between 4-tert-butylbromobenzene and 1-bromo-3-phenylpropane using $2.5 \mathrm{~mol} \% \mathrm{Co}^{\mathrm{Pc}}, 120 \mathrm{~mol} \%$ TDAE, and variable loadings of bpy $\mathrm{Ni}$ (Table 1). We selected 1,4-dioxane as the solvent due its ability to stabilize intermediates of the type $\mathrm{LNi}^{\mathrm{II}}(\mathrm{Ar}) \mathrm{X}$ (see SI), which is crucial for efficient radical capture in our envisioned pathway. Gratifyingly, we observed yields ranging from 53-84\% depending on the loading of ${ }^{\text {bpy }} \mathrm{Ni}$, with the highest yield obtained at $1 \mathrm{~mol} \%$ loading (Entry 3). Our data also highlight how the reaction can be simply optimized by varying the relative loadings of ${ }^{\text {bpy }} \mathrm{Ni}$ and $\mathrm{Co}^{\mathrm{Pc}}$. When a ${ }^{\text {bpy }} \mathrm{Ni}$ loading below $1 \mathrm{~mol} \%$ is utilized, reduced yields are obtained presumably because the rate of alkyl halide consumption is faster than the rate of aryl halide consumption as evidenced by the presence of unreacted aryl bromide when all of the alkyl bromide has been consumed (Entries 1 \& 2). In contrast, when a ${ }^{\text {bpy }} \mathrm{Ni}$ loading above $1 \mathrm{~mol} \%$ is utilized, lower yields are obtained because the rate of aryl halide consumption is faster than the rate of alkyl halide consumption as evidenced by the presence of unreacted alkyl bromide when all of the aryl bromide has been consumed (Entries 4 $\& 5)$. We suggest that these trends occur because the aryl electrophile is primarily activated by

Table 1. CEC of 4-tert-butyl-bromobenzene with 1-bromo-3-phenylpropane with varying amounts of bpyNi. a,b

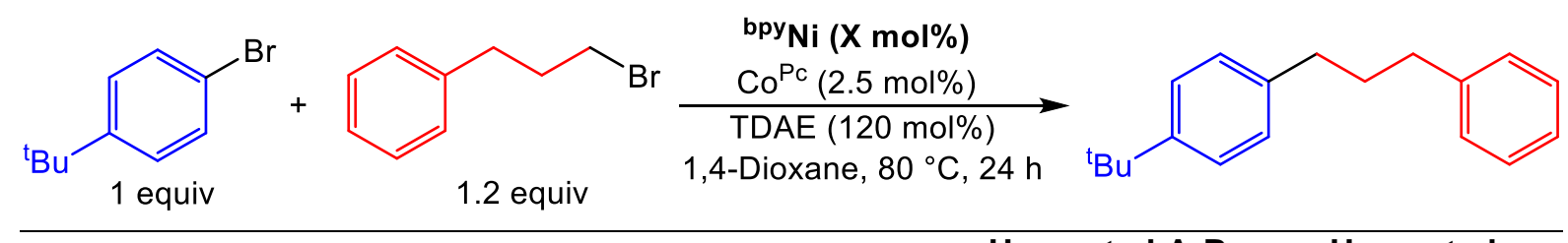

\begin{tabular}{ccccc}
\hline Entry & bpyNi (X mol\%) & Product (\%) & $\begin{array}{c}\text { Unreacted ArBr } \\
(\%)\end{array}$ & $\begin{array}{c}\text { Unreacted } \\
\text { AlkBr (\%) }\end{array}$ \\
\hline $1^{\mathrm{d}}$ & 0.1 & 55 & 29 & 3 \\
$2^{\mathrm{d}}$ & 0.5 & 68 & 22 & 6 \\
\hline 3 & 1 & 84 & 6 & $<1$ \\
\hline $4^{\mathrm{e}}$ & 2.5 & 66 & 4 & 10 \\
$5^{\dagger}$ & 5 & 53 & $<1$ & 30 \\
\hline
\end{tabular}

Reaction conditions: 1-bromo-4-tert-butylbenzene $(0.0625 \mathrm{mmol}), 1$-bromo-3-phenylpropane $(0.075 \mathrm{mmol}), \mathrm{Co}^{\mathrm{Pc}}$ $(0.0016 \mathrm{mmol})$, and TDAE $(0.075 \mathrm{mmol})$ in 1,4-dioxane $(0.5 \mathrm{~mL})$ at $80^{\circ} \mathrm{C}$ for 24 hours. bYields are reported as the average of two trials and were determined by integration of ${ }^{1} \mathrm{H}$ NMR spectra against a standard (hexamethyl

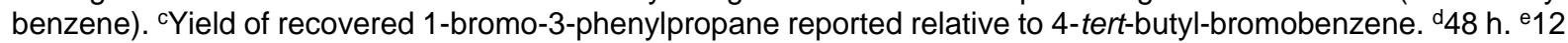
h. ${ }^{\dagger} 4 \mathrm{~h}$. 


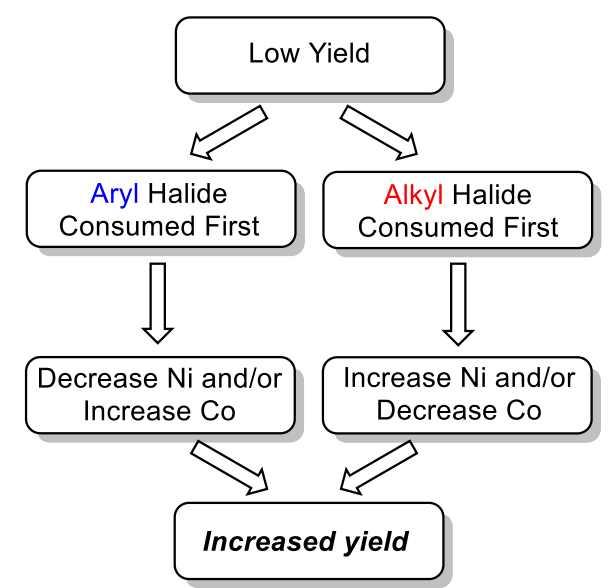

Figure 3. Optimization strategy for CEC reactions used in this work.

the Ni catalyst and the alkyl electrophile is primarily activated by the Co catalyst (vide infra). To obtain high yields, the rate of consumption of the aryl electrophile needs to be the same as the rate of consumption of the alkyl electrophile, which is accomplished by efficient alkyl radical capture at the $\mathrm{LNi}^{\mathrm{II}}(\mathrm{Ar}) \mathrm{X}$ intermediate and can be easily controlled by the loadings of ${ }^{\text {bpy }} \mathrm{Ni}$ and $\mathrm{Co}^{\mathrm{Pc}}$ (Figure 3). Further, this approach to optimization has not previously been utilized in reductive coupling reactions and provides potential advantages over methods where optimization is performed by empirical ligand and additive screening.

Using our method, we hypothesized that it would be possible to perform a diverse range of CEC reactions under the same conditions by modifying the loadings of ${ }^{\text {byy }} \mathrm{Ni}$ and $\mathrm{Co}^{\mathrm{Pc}}$ to match the reactivity of the aryl and alkyl halides, respectively. Specifically, we proposed that an aryl halide, such as iodobenzene, could be coupled with alkyl electrophiles of varying reactivities, such as unactivated alkyl iodides and highly activated benzyl chlorides, by optimizing the loadings of ${ }^{\text {bpy }} \mathrm{Ni}$ and $\mathrm{Co}^{\mathrm{Pc}}$ under the same general conditions we employed to couple aryl bromides with alkyl bromides (Scheme 1). In agreement with this model, we were able to couple iodobenzene with 1iodo-3-phenylpropane using $2.5 \mathrm{~mol} \%{ }^{\text {bpy }} \mathrm{Ni}$ and $2.5 \mathrm{~mol} \% \mathrm{Co}^{\mathrm{Pc}}$ in $97 \%$ yield under the same reaction conditions utilized in Table 1 (Scheme 1a and see SI for optimization). Further, we can also couple iodobenzene with benzyl chloride using $7 \mathrm{~mol} \%{ }^{\text {bpy }} \mathrm{Ni}$ and $0.5 \mathrm{~mol} \% \mathrm{Co}^{\mathrm{Pc}}$ in $96 \%$ yield (Scheme $1 \mathrm{~b}$ and vide infra for optimization). We propose that the reason a much higher loading of ${ }^{\text {byy }} \mathrm{Ni}$ to $\mathrm{Co}^{\mathrm{Pc}}$ is required in the coupling of benzyl chlorides is because they are more easily activated than alkyl iodides by $\mathrm{Co}^{\mathrm{Pc}}$ in catalysis. To our knowledge the ability to couple substrates with such diverse reactivity under the same general reaction conditions with high efficiency is unprecedented in $\mathrm{C}\left(\mathrm{sp}^{2}\right)-\mathrm{C}\left(\mathrm{sp}^{3}\right) \mathrm{CEC}$ (vide supra). 
Scheme 1. CEC of iodobenzene with a) 1-iodo-3-phenylpropane and b) benzyl chloride.

a)

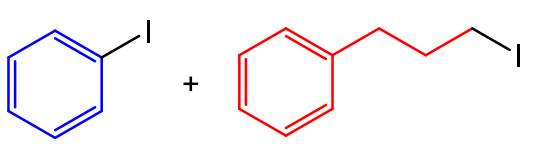

1 equiv

1.2 equiv b)<smiles>ClCc1ccccc1</smiles>

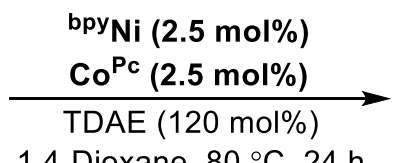

1,4-Dioxane, $80^{\circ} \mathrm{C}, 24 \mathrm{~h}$

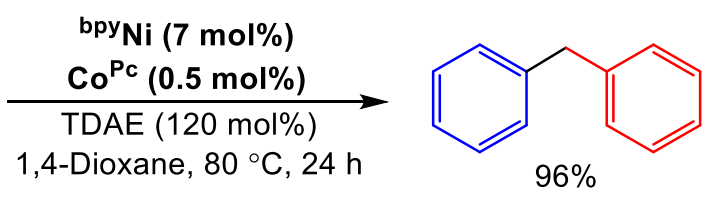

Substrate Scope for Aryl and Alkyl Bromide Coupling

Starting from the optimized conditions established for the coupling of 4-tertbutylbromobenzene and 1-bromo-3-phenylpropane we evaluated the substrate scope of the dual catalyzed method (Figure 4). Initially, we explored the coupling of a range of aryl bromides, as these are more synthetically valuable than aryl iodides, with 1-bromo-3-phenylpropane (or $N$-(3bromopropyl)phthalimide ${ }^{[17]}$ ). Our system exhibits a wide functional group tolerance, as demonstrated by the coupling of electronically diverse substrates (4a-4c) and substrates with reducible functional groups, such as nitriles (4d), sulfones (4e), aldehydes $(\mathbf{4 f})$, ketones $(\mathbf{4 g})$, esters (4h), and amides (4i). When 1-bromo-4-chlorobenzene (4j) is used as a substrate, our system is selective for coupling the aryl bromide, which offers opportunities for orthogonal reactivity with traditional cross-coupling reactions. Substrates with protic functionality, such as 4bromophenethyl alcohol (4k) and 5-bromoindole (4l) could also be coupled in high yield, although 4-bromoaniline gives a reduced yield (see $\mathrm{SI}$ ) and the more activated 4-iodoaniline (4m) is required to give a high yield. Mono-ortho-substituted substrates with moderate steric bulk (4n \& 4o) are also effectively coupled.

To show the generality and simplicity of our optimization protocol, we optimized each substrate to a yield greater than $75 \%$ by ${ }^{1} \mathrm{H}$ NMR spectroscopy using the procedure outlined in Figure 3 (see SI). ${ }^{[18]}$ Notably, the optimized conditions for each substrate deviate only slightly from our standard reaction conditions, indicating the ease by which high yields can be obtained. Further, good yields can be attained over more than an order of magnitude in ${ }^{\text {bpy }} \mathrm{Ni}$ or $\mathrm{Co}^{\mathrm{Pc}}$ loadings (Tables $1 \& 3$ ). This suggests that a wide range of substrates may be successfully coupled under a standard set of conditions even without performing the simple catalyst loading optimization. Although many of substrates 4a-4o have been coupled previously in the literature using heterogeneous $\mathrm{Mn}^{0}$ or $\mathrm{Zn}^{0}$ reductants, our system is the first to utilize a homogeneous reductant 


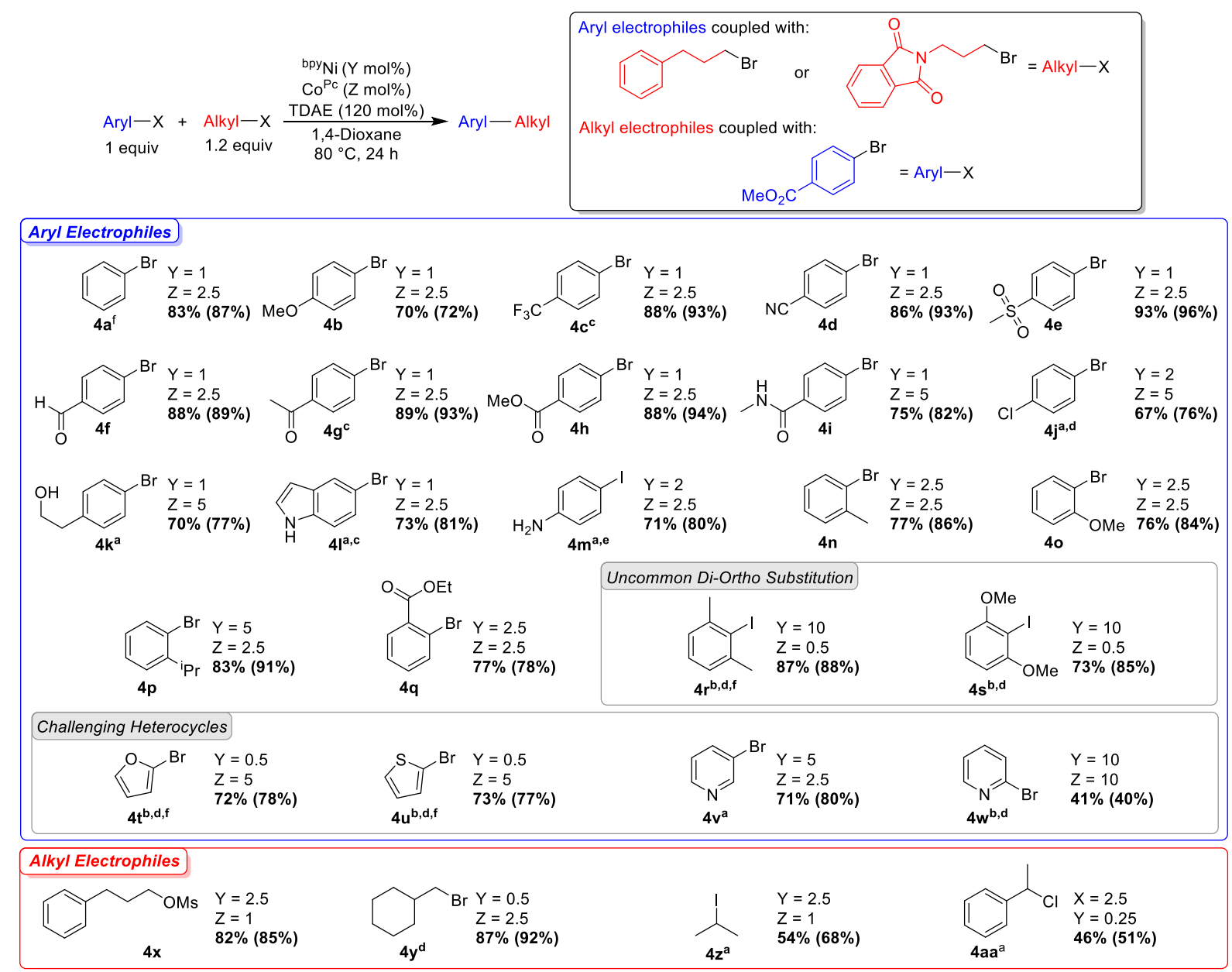

Figure 4. Substrate scope for CEC of aryl halides with alkyl halides or pseudohalides. Values outside of parentheses are isolated yields and values inside of parentheses are NMR yields, which were determined by integration of ${ }^{1} \mathrm{H}$ NMR spectra against a standard (hexamethylbenzene). ${ }^{a} 1.6$ equiv. of alkyl substrate, 140 mol\% TDAE. b2.0 equiv. of alkyl substrate, 160 mol\% TDAE. ${ }^{c} 36$ h. ${ }^{d} 48$ h. e 1 -iodo3-phenylpropane used as alkyl substrate. ${ }^{\mathfrak{N}} \mathrm{N}$-(3-bromopropyl)phthalimide used as alkyl substrate.

with such a broad substrate scope, which provides significant practical advantages (vide supra). Further, our system achieves high yields using lower than typical catalyst loadings, only uses commercially available reaction components, and does not require inscrutable additives for any of the substrates. All of these factors are advantageous for translating our results to different substrate classes.

Another significant advantage of our system is that it is compatible with some substrates that have not been traditionally used in CEC. For example, aryl halides with sterically demanding substituents in the ortho-position are a challenging class of substrates in conventional CEC. This is likely because oxidative addition of the sterically hindered aryl electrophile is difficult relative to the activation of the alkyl halide coupling partner, which results in incompatible rates of 
substrate activation in catalysis. We hypothesized that our system could overcome this challenge through modulation of catalyst loadings (vide supra). Consistent with this proposal, mono-orthosubstituted aryl bromides with significant steric bulk (4p \& 4q) could be coupled in high yield using increased loadings of ${ }^{\text {byy }} \mathrm{Ni}$ compared to reactions between bromobenzene (4a) and 1-bromo3-phenylpropane. In particular, the coupling of $\mathbf{4 q}$ is significant, as Weix et al. previously reported that it was difficult to couple aryl halides with bulky ortho-directing groups. ${ }^{[2]}$ By further increasing the loading of ${ }^{\text {by }} \mathrm{Ni}$ and decreasing the loading of $\mathrm{Co}^{\mathrm{Pc}}$, this strategy could be extended to di-ortho-substituted aryl iodides $(\mathbf{4 r} \& \mathbf{4 s})$, for which there is virtually no precedent in the CEC literature. ${ }^{[2,19]}$

Heteroaryl halides are important substrates because heteroaromatic groups are common structures in medicinal chemistry, ${ }^{[20]}$ but are traditionally difficult substrates in CEC. In particular, it has proven challenging to use 2-halofurans and 2-halothiophenes as substrates in CEC, especially when there is no substitution in the 5-position. ${ }^{[1 \mathrm{j}, 12 \mathrm{f}]}$ Our system can couple several heteroaryl substrates in high yield, including 2-bromofuran (4t), 2-bromothiophene (4u), and 3bromopyridine $(\mathbf{4 v})$. Importantly, the same optimization strategy that was utilized for simple arenes can be applied to heteroaryl substrates to overcome potential challenges associated with substrates binding to catalysts (see SI). We note, however, that we can only couple 2bromopyridine (4w) in $41 \%$ yield using $10 \mathrm{~mol} \%{ }^{\text {bpy }} \mathrm{Ni}$ and $10 \mathrm{~mol} \%$ of $\mathrm{Co}^{\mathrm{Pc}}$.

The alkyl electrophile scope was examined using the same optimization strategy that was utilized for exploring the scope of the aryl electrophile. Primary benzyl chlorides (vide supra) as well as unactivated primary alkyl iodides (vide supra) and bromides (4a-4w) can be readily coupled under our reaction conditions. However, $\mathrm{Co}^{\mathrm{Pc}}$ activates alkyl substrates via an $\mathrm{S}_{\mathrm{N}} 2$ mechanism, ${ }^{[21]}$ which differentiates it from conventional CEC systems that activate alkyl substrates through a radical pathway. ${ }^{[7 a]}$ One advantage of this difference is that our system can couple primary alkyl mesylates, such as 3-phenylpropyl methanesulfonate (4x), with methyl 4bromobenzoate in high yield. This result is notable because alkyl mesylates can be readily generated in situ from the corresponding alcohols, ${ }^{[15 b, 22]}$ which are abundant and diverse building blocks that are commonly used in pharmaceutical research. ${ }^{[14 a]}$ In contrast, while substrates with some steric bulk at the $\alpha$-carbon of alkyl bromides, such as (bromomethyl)cyclohexane (4y), can be coupled with methyl 4-bromobenzoate, no product is generated when either neopentyl bromide or iodide are used as substrates (see SI). Similarly, branched secondary alkyl halides such as 
iodides $(\mathbf{4 z})$ and benzyl chlorides (4aa) can be coupled in moderate yields, but branched secondary alkyl bromides and iodocyclohexane are unreactive (see SI). In a subsequent section we explore the types of functionalized alkyl bromides and iodides and benzyl chlorides that are compatible with our system.

\section{Applications to Medicinal Chemistry}

Despite the significant attention that $\mathrm{C}\left(\mathrm{sp}^{2}\right)-\mathrm{C}\left(\mathrm{sp}^{3}\right)$ cross-electrophile reactions have received over the past decade, it remains difficult to translate this methodology to complex, medicinally relevant substrates. ${ }^{[6,23]}$ Given the high value of compounds containing alkylated arene groups in the development and study of pharmaceuticals, a robust and generalizable method to form $\mathrm{C}\left(\mathrm{sp}^{2}\right)-\mathrm{C}\left(\mathrm{sp}^{3}\right)$ linkages with medicinally relevant substrates would be valuable for drug discovery. ${ }^{[24]}$ To this end, we tested the compatibility of our reaction conditions with aryl halides from the MSD Aryl Halide Informer Library, as these compounds were at one time intermediates in drug discovery programs. ${ }^{[25]}$ Using standard high-throughput experimentation (HTE) techniques, we were able to rapidly optimize the reactions by varying the catalyst loadings for eight substrates in a single experiment using a standard 96 well reaction plate (see SI). ${ }^{[26]}$ Aryl halides $\mathbf{5 a - 5 h}$ were successfully coupled in moderate to high yields (42-91\% yield by ${ }^{1} \mathrm{H}$ NMR spectroscopy) with 1-bromo-3-phenylpropane using catalyst loadings between 0.5 and $5 \mathrm{~mol} \%$ of both ${ }^{\text {byy }} \mathrm{Ni}$ and $\mathrm{Co}^{\mathrm{Pc}}$ without altering any other reaction parameters (Figure 5). The range of functional groups present in these aryl halides highlights the power of our method. For example, successful reactions were observed in the presence of esters, amides, sulfones, alcohols, triazoles, thiophenes, pyridines, and both free and protected amines among many other functional groups. Notably, the challenging di-ortho substituted aryl halide containing a pendant primary amine, $\mathbf{5 i}$, was coupled in lower, but still medicinally useful, yield (22\% by ${ }^{1} \mathrm{H}$ NMR spectroscopy) with 1 iodo-3-phenylpropane. Additionally, we isolated the product from the reaction of 5a with 1bromo-3-phenylpropane in good yield $(72 \%)$ and demonstrated that the reaction is scalable through a coupling using $3 \mathrm{mmol}$ of $\mathbf{5 f}$ (see SI) as proof-of-principle that our method will enable the generation of compounds for drug discovery.

To further investigate the potential applicability of our reaction conditions to $\mathrm{C}\left(\mathrm{sp}^{2}\right)-\mathrm{C}\left(\mathrm{sp}^{3}\right)$ bond formation in molecules relevant to drug discovery, we performed a parallel library synthesis via late-stage diversification of aryl halide $\mathbf{5 f}$, a precursor to oxazolidinone antibacterials, with 

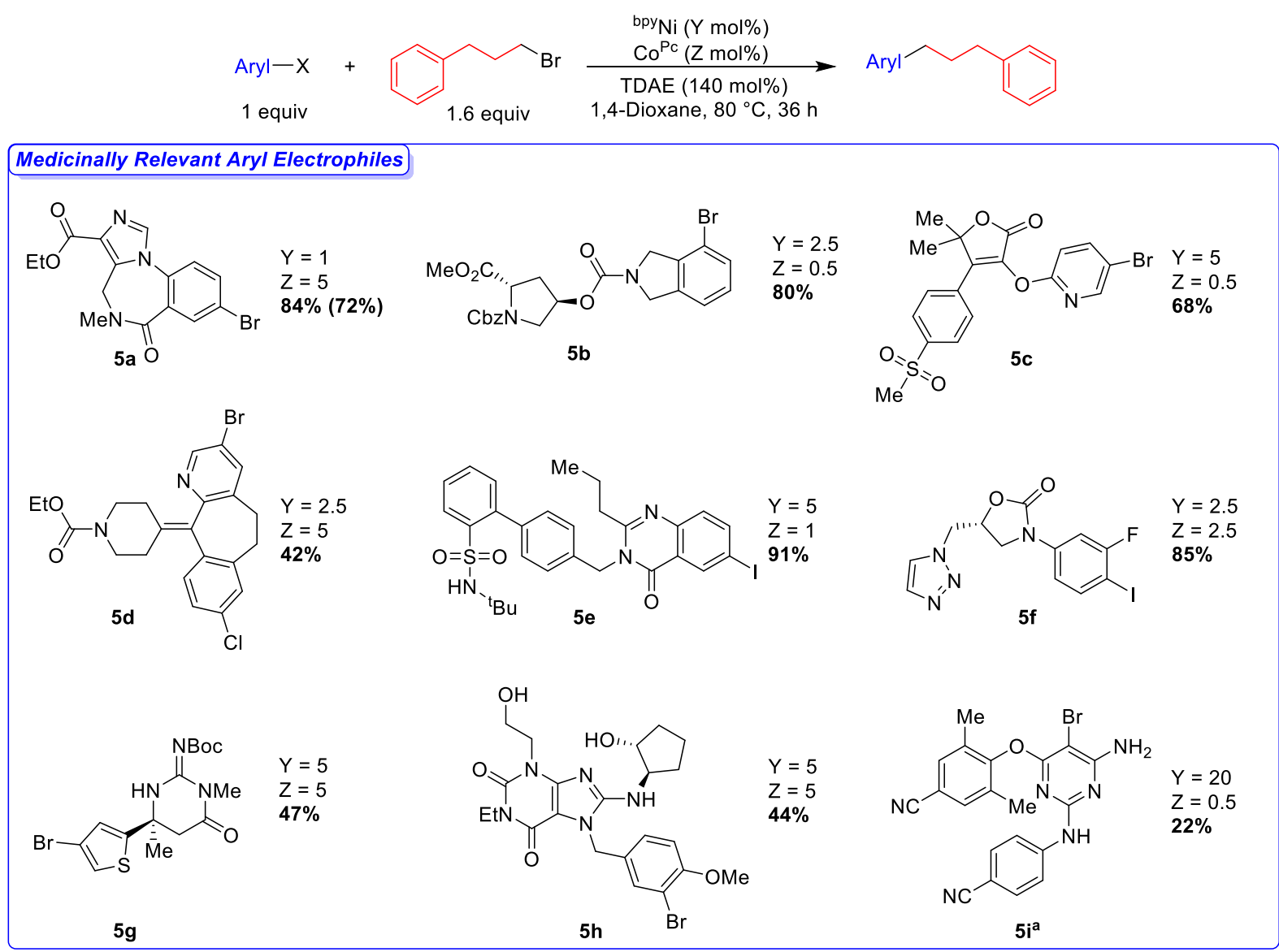

Figure 5. Substrate scope for CEC between examples from the MSD Aryl Halide Informer Library and 1-bromo-3-phenylpropane. Values outside of parentheses are NMR yields, which were determined by integration of ${ }^{1} \mathrm{H}$ NMR spectra against a standard (hexamethylbenzene), and values inside of parentheses are isolated yields. ${ }^{2} 2$ equivalents of 1-iodo-3-phenylpropane alkyl substrate, $160 \mathrm{~mol} \%$ TDAE, $48 \mathrm{~h}$.

different alkyl halides (Figure 6). ${ }^{[27]}$ First, we optimized the loadings of ${ }^{\text {bpy }} \mathrm{Ni}$ and $\mathrm{Co}^{\mathrm{Pc}}$ for the reactions of $\mathbf{5 f}$ with a benzylic chloride, an alkyl iodide, and an alkyl bromide (see SI). We then used the optimized conditions for each class of alkyl halide to evaluate the coupling of a series of functionalized derivatives with $\mathbf{5 f}$ using HTE techniques (see SI). For example, all primary alkyl bromides used in the experiment were coupled under the optimal conditions determined for the coupling of $\mathbf{5 f}$ with 1-bromo-3-phenylpropane (6r). Using our strategy, we observed that 25 out of 32 products were formed in greater than $10 \%$ conversion, an overall $78 \%$ success rate. A range of functionalized primary benzyl chloride electrophiles could be coupled, including substrates containing a tetrazole $(\mathbf{6 b})$ or thiophene (6d) ring, or a protic amide substituent (6f). Further, various primary alkyl iodide and bromide electrophiles could be coupled, such as substrates containing an unprotected indole (6o) and terminal alkenes $(\mathbf{6 x})$, which are susceptible to Giese- 

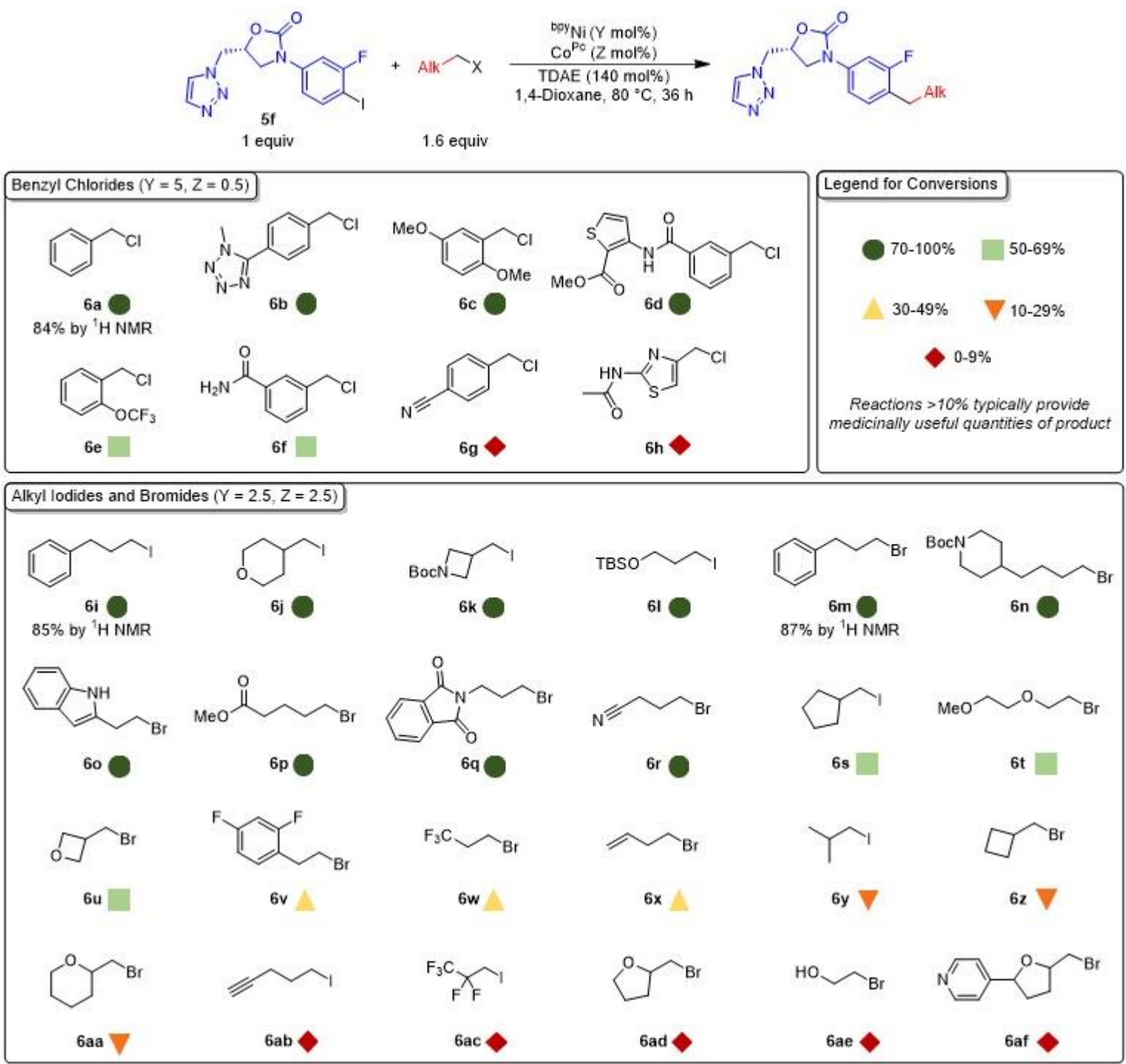

Figure 6. CEC between $\mathbf{5 f}$ and a series of benzylic chlorides, alkyl iodides, and alkyl bromides. Values are reported as the conversion to product relative to all known species derived from $\mathbf{5} \mathbf{f}$ determined by UV-Visible spectroscopy (see SI for details). NMR yields were determined by integration of ${ }^{1} \mathrm{H}$ NMR spectra against a standard (hexamethylbenzene).

type additions in related reactions. ${ }^{[16 \mathrm{~b}, 16 \mathrm{c}]}$ Additionally, heterocyclic rings, which are prevalent in medicinal chemistry, such as azetidine (6k), piperidine (6n), and cyclic ethers $(\mathbf{6 j}, \mathbf{6} \mathbf{u}, \mathbf{6 a a})$ are compatible with our method. ${ }^{[28]}$ Overall, this experiment shows that our methodology can tolerate a large number of functional groups on the alkyl halide substrate. It also shows that the optimized reactions conditions obtained for an individual aryl halide substrate can be readily translated to a broad range of alkyl halides without reoptimization, which enables efficient parallel library synthesis and should be valuable in medicinal chemistry.

Apart from the improved substrate scope, our system offers practical advantages for 
performing CEC reactions in a medicinal chemistry setting compared to existing methodology. (i) Experimental setup is straightforward because all reactions utilize the same reaction components under the same reaction conditions. In contrast, conventional CEC protocols operate within an almost indeterminate optimization space as various reaction parameters, such as ancillary ligand, solvent, and inscrutable additives, are all typically individually optimized for each substrate. (ii) It is compatible with a wide range of solvents, including green solvents (see SI), which assists in finding conditions where substrates are fully soluble and reduces environmental impact. ${ }^{[29]}$ (iii) It utilizes a homogeneous reductant as opposed to a heterogeneous reductant, which is important for scale-up.

\section{Three-Component Coupling Reactions}

Reactions that enable the modification of simple aryl rings in a modular fashion are valuable for the creation of diverse libraries of compounds, which often facilitate the discovery of lead structures in medicinal chemistry. ${ }^{[30]}$ To this end, readily accessible dihaloarenes represent promising starting materials from which to directly and efficiently construct a wide range of structures. ${ }^{[31]}$ Although there are currently numerous methods for the sequential introduction of aryl groups into dihaloarenes via standard $\mathrm{C}\left(\mathrm{sp}^{2}\right)-\mathrm{C}\left(\mathrm{sp}^{2}\right)$ cross-coupling reactions, ${ }^{[30-32]}$ there is only a single report that demonstrates the sequential introduction of alkyl groups, ${ }^{[33]}$ and no reports that utilize CEC. Using our method, we performed one-pot three-component CEC reactions involving the sequential addition of two alkyl halides to an iodo(bromo)arene (Table 2). These reactions take advantage of the increased reactivity of iodoarenes over bromoarenes. Initially, upon completion of a reaction between 1-bromo-4-iodo-2-methoxybenzene and benzyl chloride to selectively form the monoalkylated bromoarene product, we added ethyl 4-bromobutyrate and TDAE and continued the reaction. The in situ generated bromoarene product underwent a second CEC with the alkyl bromide, without the need to add additional amounts of either catalyst, suggesting that there is no catalyst death either during or upon completion of the initial alkylation (Entry 1, see SI for optimization). Across the two steps, the isolated yield for the bis-alkylated product was $82 \%$. Under the same reaction conditions, 1-bromo-2-iodo-4-methylbenzene can also be coupled with benzyl chloride and ethyl 4-bromobenzoate in $91 \%$ yield (Entry 2).

Using the sequential addition strategy, reactions that require different catalyst loadings for the first and second coupling can be performed by introducing additional equivalents of either catalyst after the completion of the initial coupling. Through this method, 1-iodo-3-phenylpropane 
Table 2. One-pot three-component CEC of bromo(iodo)arenes with alkyl halides. ${ }^{a}$

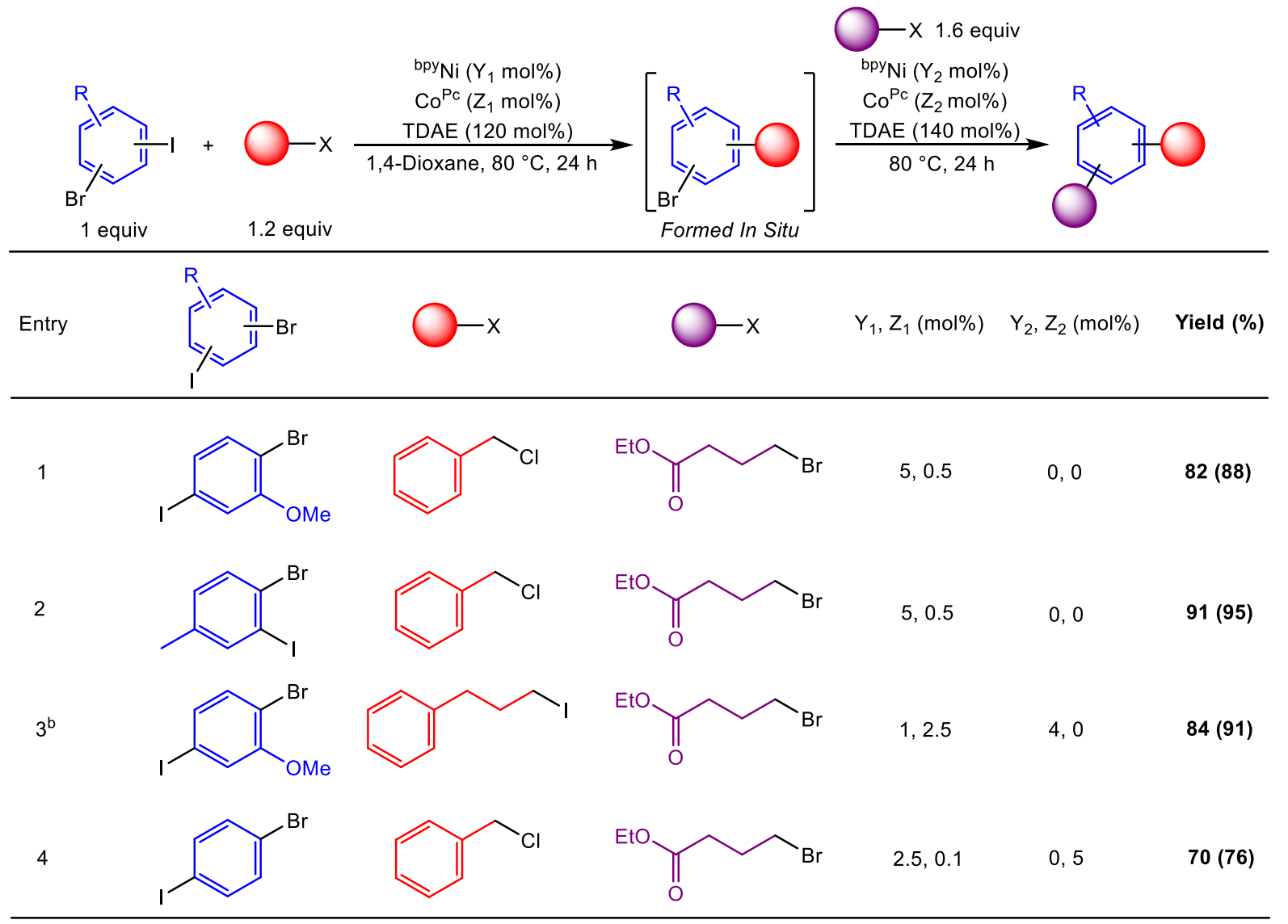

aYields outside of parentheses are isolated yields and yields inside of parentheses are NMR yields, which were determined by integration of ${ }^{1} \mathrm{H}$ NMR spectra against a standard (hexamethylbenzene). ${ }^{\mathrm{b}} 1.1$ equivalents of 1 -iodo-3-phenylpropane and 110 mol\% TDAE used in initial coupling.

and ethyl 4-bromobutyrate could be sequentially coupled with 1-bromo-4-iodo-2-methoxybenzene in $84 \%$ yield through the addition of 4 mol\% ${ }^{\text {bpy }} \mathrm{Ni}$ after the initial coupling (Entry 3 ). Additionally, an unsubstituted bromo(iodo)arene, 1-bromo-4-iodobenzene, was coupled with benzyl chloride and ethyl 4-bromobenzoate in $70 \%$ yield through the addition of $5 \mathrm{~mol} \% \mathrm{Co}^{\mathrm{Pc}}$ after the initial coupling (Entry 4). Further, in some cases, it is possible to perform these transformations in a single-step, as demonstrated by the coupling of 1-bromo-4-iodo-2-methoxybenzene with benzyl chloride and 1-bromo-3-phenylpropane with high regioselectivity to generate the desired product in 76\% yield (see Eq 1 and SI for further details), which extends the practicality of the method. The examples presented here serve as proof-of-principle that our dual catalytic platform can be used for the one-pot construction of multiple $\mathrm{C}\left(\mathrm{sp}^{2}\right)-\mathrm{C}\left(\mathrm{sp}^{3}\right)$ bonds using readily available bromo(iodo)arene and alkyl halide starting materials and highlight the potential utility of our CEC strategy towards the discovery of novel reactions. Our method is a significant advancement over 
existing methodology for dialkylation of bromo(iodo)arenes, which cannot be performed in onepot and require the use of preformed organometallic nucleophiles, such as alkylbis(catecholato)silicates, that are not commercially available and generally require multistep syntheses. ${ }^{[33]}$

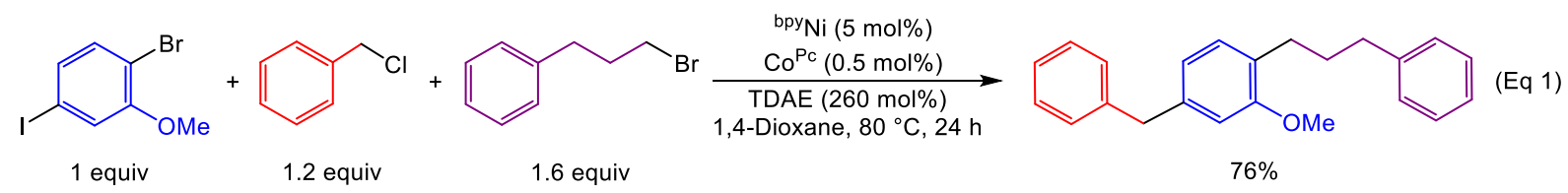

Preliminary Mechanistic Investigation

To gain further insight into the reaction mechanism, we explored a CEC reaction between the activated substrates phenyl iodide and benzyl chloride (Table 3). In the absence of $\mathrm{Co}^{\mathrm{Pc}}$, the reaction proceeded in $40 \%$ yield (Entry 1). Significant quantities of benzyl chloride were still present at the end of the reaction, showing that alkyl halide consumption is slow relative to aryl halide consumption. Notably, $\mathrm{Ni}$ is able to engage the alkyl electrophile to promote catalysis in the absence of $\mathrm{Co}^{\mathrm{Pc}}$ with the highly activated benzyl chloride substrate. In contrast, when reactions are performed with weakly activated alkyl halides, such as primary alkyl bromides, no product formation is observed in the absence of $\mathrm{Co}^{\mathrm{Pc}}$ (see SI). These observations align with our hypothesis and suggest that ${ }^{\text {byy }} \mathrm{Ni}$ primarily activates the aryl electrophile, while $\mathrm{Co}^{\mathrm{Pc}}$ primarily activates the alkyl electrophile.

The addition of varying amounts of $\mathrm{Co}^{\mathrm{Pc}}$ into the reaction results in clear trends, which mirror those observed in Table 1. We propose that the reaction can be broken down into three distinct regimes which are related to the relative loadings of ${ }^{\text {bpy }} \mathrm{Ni}$ and $\mathrm{Co}^{\mathrm{Pc}}$. Regime 1 occurs when the rate of radical formation and capture at $(\mathrm{dtbbpy}) \mathrm{Ni}^{\mathrm{II}}(\mathrm{Ar}) \mathrm{X}$ is slow relative to the rate of decomposition of (dtbbpy) $\mathrm{Ni}^{\mathrm{II}}(\mathrm{Ar}) \mathrm{X}$, which deleteriously consumes the aryl halide to produce biaryl and protodehalogenated products (Figure 2). ${ }^{[12 c]}$ Further, unreacted alkyl halide remains after all of the aryl electrophile has been consumed (Entry 2). Regime 2 occurs when the rate of alkyl radical formation and capture at (dtbbpy) $\mathrm{Ni}^{\mathrm{II}}(\mathrm{Ar}) \mathrm{X}$ is optimal relative to the formation and decomposition of (dtbbpy) $\mathrm{Ni}^{\mathrm{II}}(\mathrm{Ar}) \mathrm{X}$. In this regime, high product yields are observed and no unreacted starting material remains at the end of the reaction (Entries 3-6). Regime 3 occurs when alkyl radical formation is faster than the generation of (dtbbpy) $\mathrm{Ni}^{\mathrm{II}}(\mathrm{Ar}) \mathrm{X}$, and, as a result, the alkyl radical decomposes before it can be trapped by $\mathrm{Ni}$ and unreacted aryl halide remains after the alkyl halide has been consumed (Entries $7 \& 8$ ). The same trends are also obtained when the loading of 
${ }^{\text {bpy }} \mathrm{Ni}$ is varied at a fixed loading of $\mathrm{Co}^{\mathrm{Pc}}$ in the coupling of iodobenzene with benzyl chloride (see SI).

Table 3. CEC of iodobenzene and benzyl chloride with varying amounts of $\mathrm{Co}^{\mathrm{Pc}} . \mathrm{a}, \mathrm{b}$

\begin{tabular}{|c|c|c|c|c|c|c|}
\hline & & 1.2 equiv & $\begin{array}{r}\text { bpy Ni }\left(7^{\mathbf{C o}^{\mathrm{Pc}}(\mathbf{X}}\right. \\
\text { TDAE }(12 \\
\text { 1,4-Dioxane, }\end{array}$ & $\begin{array}{l}\underset{\mathrm{Dol} \%)}{\mathrm{ol} \%)} \\
\underset{{ }^{\circ} \mathrm{C}, 24 \mathrm{~h}}{\longrightarrow}\end{array}$ & & \\
\hline Entry & $\begin{array}{c}\mathrm{Co}^{\mathrm{Pc}} \\
(\mathrm{X} \text { mol\%) }\end{array}$ & $\begin{array}{c}\text { Product } \\
(\%)\end{array}$ & $\begin{array}{c}\text { Unreacted } \\
\text { Arl (\%) }\end{array}$ & $\begin{array}{c}\text { Biphenyl } \\
(\%)\end{array}$ & $\begin{array}{l}\text { Unreacted } \\
\mathrm{BnCl}(\%)^{\mathrm{c}}\end{array}$ & $\begin{array}{c}\text { Catalytic } \\
\text { Regime }\end{array}$ \\
\hline $\begin{array}{l}1 \\
2\end{array}$ & $\begin{array}{c}0 \\
0.01\end{array}$ & $\begin{array}{l}40 \\
77\end{array}$ & $\begin{array}{c}3 \\
<1\end{array}$ & $\begin{array}{c}21 \\
6\end{array}$ & $\begin{array}{l}28 \\
16\end{array}$ & 1 \\
\hline $\begin{array}{l}3 \\
4 \\
5 \\
6\end{array}$ & $\begin{array}{c}0.1 \\
0.25 \\
0.5 \\
1\end{array}$ & $\begin{array}{l}90 \\
87 \\
96 \\
90\end{array}$ & $\begin{array}{l}5 \\
4 \\
3 \\
4\end{array}$ & $\begin{array}{l}2 \\
2 \\
1 \\
2\end{array}$ & $\begin{array}{l}4 \\
<1 \\
<1 \\
<1\end{array}$ & 2 \\
\hline $\begin{array}{l}7 \\
8\end{array}$ & $\begin{array}{c}2.5 \\
5\end{array}$ & $\begin{array}{l}75 \\
62\end{array}$ & $\begin{array}{l}14 \\
21\end{array}$ & $\begin{array}{l}1 \\
1\end{array}$ & $\begin{array}{l}<1 \\
<1\end{array}$ & 3 \\
\hline
\end{tabular}

aReaction conditions: iodobenzene $(0.0625 \mathrm{mmol})$, benzyl chloride $(0.075 \mathrm{mmol})$, by $\mathrm{Ni}(0.0044 \mathrm{mmol})$, and TDAE $(0.075 \mathrm{mmol})$ in 1,4 -dioxane $(0.5 \mathrm{~mL})$ at $80^{\circ} \mathrm{C}$ for $24 \mathrm{~h}$. bYields are reported as the average of two trials and were determined by integration of ${ }^{1} \mathrm{H}$ NMR spectra against a standard (hexamethylbenzene). Yield of recovered benzyl chloride reported relative to iodobenzene loading.

To further explore the proposed interplay between $\mathrm{Ni}$ and $\mathrm{Co}$ in catalysis, we performed a stoichiometric reaction between (dtbbpy) $\mathrm{Ni}^{\mathrm{II}}(o$-tol $) \mathrm{I}$ and benzyl chloride in the presence of excess TDAE and catalytic amounts of $\mathrm{Co}^{\mathrm{Pc}}$ (Table 4). This resulted in the generation of the diarylmethane cross-product, (o-tolyl)(phenyl)methane, in 76\% yield (Entry 1$)$. No product formation, however, was observed without $\mathrm{Co}^{\mathrm{Pc}}$ in either the presence or absence of TDAE (Entries $2 \& 3$ ), consistent with our hypothesis that Co primarily activates the alkyl electrophile in catalysis. Furthermore, the use of stoichiometric $\mathrm{Co}^{\mathrm{Pc}}$ without TDAE also yielded no cross-product (Entry 4), suggesting that the activation of alkyl electrophiles occurs at a reduced Co center. In agreement with this proposal, the reduction potential of $\mathrm{TDAE}^{2+/ 0}$ is more negative than that of the $\mathrm{Co}^{\mathrm{II} / \mathrm{I}}$ couple of $\mathrm{Co}^{\mathrm{Pc}} \cdot{ }^{[34]} \mathrm{Low}-$ valent Co complexes similar to $\left[\mathrm{Co}^{\mathrm{I}}(\mathrm{Pc})\right]^{-}$are known to undergo oxidative addition with alkyl halides through an $\mathrm{S}_{\mathrm{N}} 2$ mechanism to form $\mathrm{Co}^{\mathrm{III}}(\mathrm{Pc})(\mathrm{Alk})$ species. ${ }^{[21]}$ In turn, these high valent $\mathrm{Co}^{\mathrm{III}}$ complexes can undergo homolysis of the $\mathrm{Co}^{\mathrm{III}}-\mathrm{Alk}$ bond, which produces an alkyl radical and regenerates $\mathrm{Co}^{\mathrm{Pc}} \cdot{ }^{[35]}$ Further support for the proposal that $\mathrm{Co}^{\mathrm{Pc}}$ is capable of generating alkyl radicals in the presence of TDAE was obtained by performing an analogous radical trapping experiment using 2,2,6,6-tetramethyl-1-piperidinyloxy (TEMPO) as the radical acceptor instead of (dtbbpy)Ni ${ }^{\mathrm{II}}\left(o\right.$-tol)I. ${ }^{[36]}$ In a similar fashion to our experiment with (dtbbpy) $\mathrm{Ni}^{\mathrm{II}}(o$-tol)I, trapping 
Table 4. Reaction of (dtbbpy)Ni"(o-tol)I with benzyl chloride under various conditions. ${ }^{a, b}$

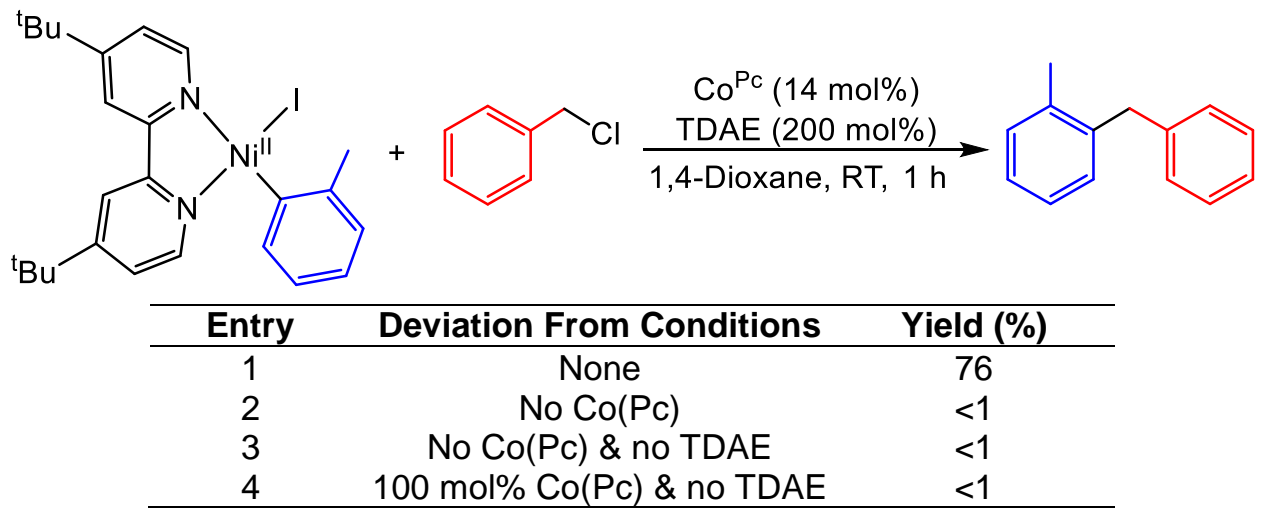

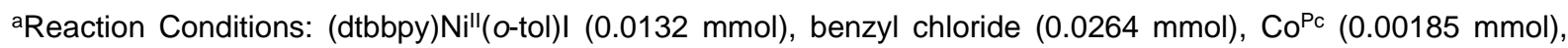
$\operatorname{TDAE}(0.0264 \mathrm{mmol})$, in 1,4 -dioxane $(1.5 \mathrm{~mL})$ at $\mathrm{RT}$ for $1 \mathrm{~h}$. ${ }^{\text {bY }}$ ields are reported as the average of two trials and were determined by integration of ${ }^{1} \mathrm{H}$ NMR spectra against a standard (hexamethylbenzene).

of the benzyl radical by TEMPO is only observed in the presence of excess TDAE and catalytic amounts of $\mathrm{Co}^{\mathrm{Pc}}$ (see SI). Altogether, these experiments provide evidence for the Co-mediated generation of free radicals from an alkyl electrophile and subsequent radical capture by (dtbbpy) $\mathrm{Ni}^{\mathrm{II}}(\mathrm{Ar}) \mathrm{X}$ species in catalysis.

On the basis of our experimental results, we propose a mechanism containing two cycles for the coupling of phenyl iodide and benzyl chloride catalyzed by ${ }^{\text {bpy }} \mathrm{Ni}$ and $\mathrm{Co}^{\mathrm{Pc}}$ (Figure 7). Initially, the ${ }^{\text {bpy }} \mathrm{Ni}$ precatalyst is reduced by TDAE to generate a catalytically active $\mathrm{Ni}^{0} \mathrm{species}$ The $\mathrm{Ni}^{0}$ species undergoes oxidative addition with an aryl halide to form a (dtbbpy)Ni ${ }^{\mathrm{II}}(\mathrm{Ar}) \mathrm{X}$ intermediate, which is likely the resting state of the $\mathrm{Ni}$ catalyst. Subsequently, the (dtbbpy) $\mathrm{Ni}^{\mathrm{II}}(\mathrm{Ar}) \mathrm{X}$ intermediate captures an alkyl radical, which is liberated upon the homolysis of

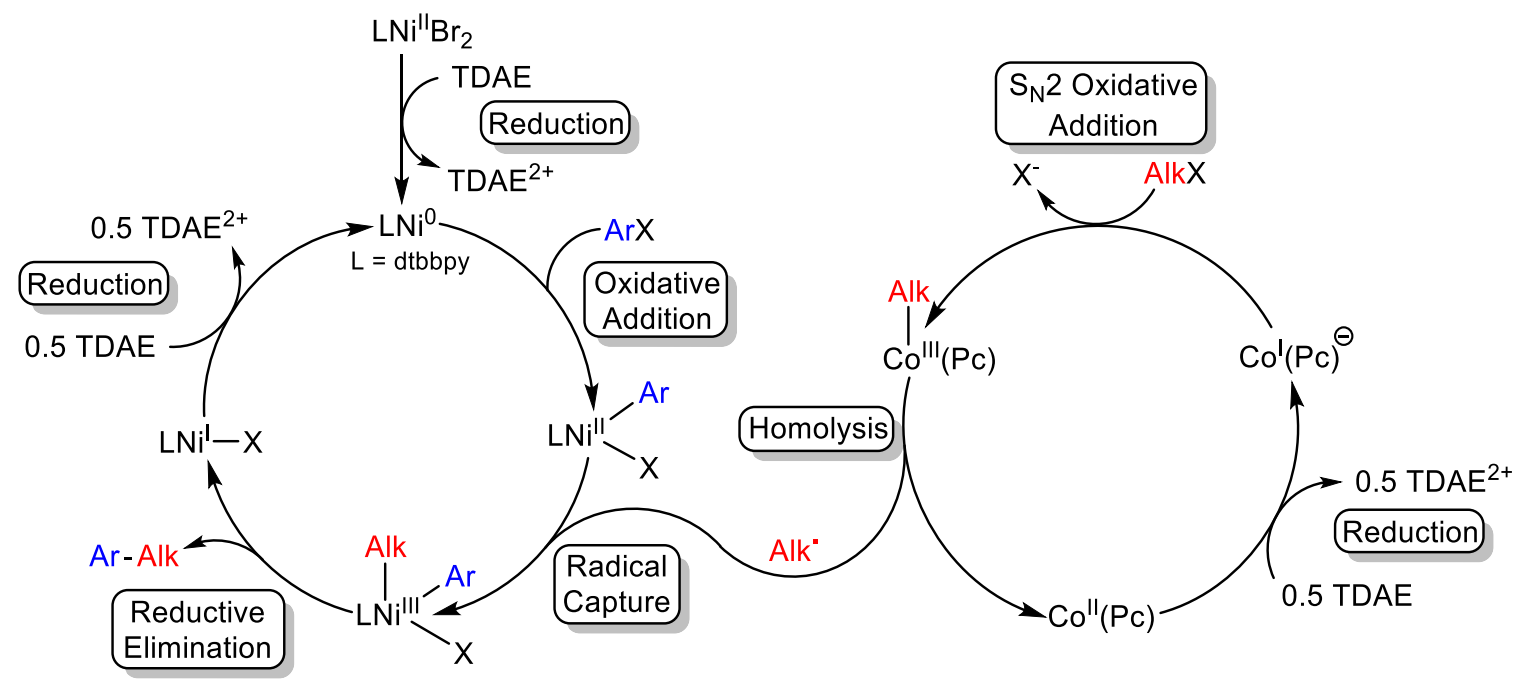

Figure 7. Proposed mechanism for the CEC of aryl and alkyl halides in the presence of $\mathrm{Co}^{\mathrm{Pc}}$ and TDAE. 
a $\mathrm{Co}^{\mathrm{III}}(\mathrm{Pc})(\mathrm{Alk})$ species. The $\mathrm{Co}^{\mathrm{III}}(\mathrm{Pc})(\mathrm{Alk})$ species is generated in an independent catalytic cycle through initial reduction of $\mathrm{Co}^{\mathrm{Pc}}$ to form an anionic $\left[\mathrm{Co}^{\mathrm{I}}(\mathrm{Pc})\right]^{-}$complex, which can react with an alkyl halide via an $\mathrm{S}_{\mathrm{N}} 2$ mechanism. Following radical capture at (dtbbpy) $\mathrm{Ni}^{\mathrm{II}}(\mathrm{Ar}) \mathrm{X}$, a putative (dtbbpy) $\mathrm{Ni}^{\mathrm{III}}(\mathrm{Ar})(\mathrm{Alk}) \mathrm{X}$ species is produced, which rapidly reductively eliminates at the $\mathrm{Ni}^{\mathrm{III}}$ center to liberate the product and form a (dtbbpy) $\mathrm{Ni}^{\mathrm{I} X}$ species. Finally, we propose that the (dtbbpy) $\mathrm{Ni}^{\mathrm{I} X}$ species is reduced by TDAE to regenerate $\mathrm{Ni}^{0}$, closing the catalytic cycle. Further mechanistic work to explore all of the potential roles of $\mathrm{Ni}^{\mathrm{I}}$ species is ongoing.

\section{Conclusions}

We have developed a novel system for $\mathrm{C}\left(\mathrm{sp}^{2}\right)-\mathrm{C}\left(\mathrm{sp}^{3}\right) \mathrm{CEC}$ reactions of aryl and alkyl halides. Our system uses a $\mathrm{Ni}$ and $\mathrm{Co}$ dual catalytic platform in tandem with a relatively weak homogenous reductant to ensure that the key $\mathrm{LNi}^{\mathrm{II}}(\mathrm{Ar}) \mathrm{X}$ intermediate undergoes on-cycle reactions. Our system is able to efficiently couple a wide a range of substrates including heteroaryl halides, di-ortho-substituted aryl iodides, drug-like aryl halides and functionally diverse alkyl halides, all of which are rarely compatible with traditional methods for $\mathrm{C}\left(\mathrm{sp}^{2}\right)-\mathrm{C}\left(\mathrm{sp}^{3}\right) \mathrm{CEC}$. Additionally, we are able to perform a series of novel one-pot, three-component dialkylations of bromo(iodo)arenes. Given the importance of $\mathrm{LNi}^{\mathrm{II}}(\mathrm{Ar}) \mathrm{X}$ intermediates in Ni-catalyzed radical coupling reactions, we suggest that our strategy of selecting reaction conditions that stabilize this complex and allow for controlled generation of alkyl radicals are relevant to a large number of other reactions.

\section{Acknowledgements}

NH acknowledges support from the NIHGMS under Award Number R01GM120162. We thank Professor Jon Ellman and Dr. Louis-Charles Campeau for valuable discussions. We also thank Sr. Scientist Scott Borges, May Ann Desaca, and Sr. Scientist Tao Meng for purification support, Assoc. Scientist Ming Wang for assistance with high-throughput analytical sample processing, and Dr. Fabian Menges for help with mass spectrometry.

\section{Supporting information}

Additional information about selected experiments, NMR spectra, and other details are available via the Internet.

\section{References}


[1] For leading references, see: (a) D. A. Everson, R. Shrestha, D. J. Weix, J. Am. Chem. Soc. 2010, 132, 920 ; (b) D. A. Everson, B. A. Jones, D. J. Weix, J. Am. Chem. Soc. 2012, 134, 6146; (c) A. C. Wotal, D. J. Weix, Org. Lett. 2012, 14, 1476; (d) D. A. Everson, D. J. Weix, J. Org. Chem. 2014, 79, 4793; (e) C. Zhao, X. Jia, X. Wang, H. Gong, J. Am. Chem. Soc. 2014, 136, 17645; (f) J. Gu, X. Wang, W. Xue, H. Gong, Org. Chem. Front. 2015, 2, 1411; (g) D. J. Weix, Acc. Chem. Res. 2015, 48, 1767; (h) Y. Zhao, D. J. Weix, J. Am. Chem. Soc. 2015, 137, 3237; (i) K. M. Arendt, A. G. Doyle, Angew. Chem. Int. Ed. 2015, 54, 9876; (j) N. T. Kadunce, S. E. Reisman, J. Am. Chem. Soc. 2015, 137, 10480; (k) G. A. Molander, K. M. Traister, B. T. O’Neill, J. Org. Chem. 2015, 80, 2907; (1) X. Wang, S. Wang, W. Xue, H. Gong, J. Am. Chem. Soc. 2015, 137, 11562; (m) L. Hu, X. Liu, X. Liao, Angew. Chem. Int. Ed. 2016, 55, 9743; (n) K. A. Johnson, S. Biswas, D. J. Weix, Chem. Eur. J. 2016, 22, 7399; (o) M. O. Konev, L. E. Hanna, E. R. Jarvo, Angew. Chem. Int. Ed. 2016, 55, 6730; (p) F. Chen, K. Chen, Y. Zhang, Y. He, Y.-M. Wang, S. Zhu, J. Am. Chem. Soc. 2017, 139, 13929; (q) K. E. Poremba, N. T. Kadunce, N. Suzuki, A. H. Cherney, S. E. Reisman, J. Am. Chem. Soc. 2017, 139, 5684; (r) B. P. Woods, M. Orlandi, C.-Y. Huang, M. S. Sigman, A. G. Doyle, J. Am. Chem. Soc. 2017, 139, 5688; (s) P. Schneider, G. Schneider, Angew. Chem. Int. Ed. 2017, 56, 7971; (t) L. Peng, Y. Li, Y. Li, W. Wang, H. Pang, G. Yin, ACS Catal. 2018, 8, 310; (u) X. Wang, G. Ma, Y. Peng, C. E. Pitsch, B. J. Moll, T. D. Ly, X. Wang, H. Gong, J. Am. Chem. Soc. 2018, 140, 14490; (v) S. Ni, C.-X. Li, Y. Mao, J. Han, Y. Wang, H. Yan, Y. Pan, Sci. Adv. 2019, 5, 9516; (w) Y. Ye, H. Chen, J. L. Sessler, H. Gong, J. Am. Chem. Soc. 2019, 141, 820; (x) H. Yue, C. Zhu, L. Shen, Q. Geng, K. J. Hock, T. Yuan, L. Cavallo, M. Rueping, Chem. Sci. 2019, 10, 4430.

[2] E. C. Hansen, D. J. Pedro, A. C. Wotal, N. J. Gower, J. D. Nelson, S. Caron, D. J. Weix, Nature Chem. 2016, 8, 1126.

[3] E. S. Isbrandt, R. J. Sullivan, S. G. Newman, Angew. Chem. Int. Ed. 2019, 58, 7180.

[4] (a) C.-S. Yan, Y. Peng, X.-B. Xu, Y.-W. Wang, Chem. Eur. J. 2012, 18, 6039; (b) G. A. Molander, K. M. Traister, B. T. O’Neill, J. Org. Chem. 2014, 79, 5771; (c) L. L. Anka-Lufford, K. M. M. Huihui, N. J. Gower, L. K. G. Ackerman, D. J. Weix, Chem. Eur. J. 2016, 22, 11564; (d) J. Zhang, G. Lu, J. Xu, H. Sun, Q. Shen, Org. Lett. 2016, $18,2860$.

[5] E. C. Hansen, C. Li, S. Yang, D. Pedro, D. J. Weix, J. Org. Chem. 2017, 82, 7085.

[6] (a) J. M. E. Hughes, P. S. Fier, Org. Lett. 2019, 21, 5650; (b) K. M. Mennie, B. A. Vara, S. M. Levi, Org. Lett. 2020, 22, 556.

[7] (a) S. Biswas, D. J. Weix, J. Am. Chem. Soc. 2013, 135, 16192; (b) J. B. Diccianni, J. Katigbak, C. Hu, T. Diao, J. Am. Chem. Soc. 2019, 141, 1788; (c) Q. Lin, T. Diao, J. Am. Chem. Soc. 2019, 141, 17937; (d) M. Mohadjer Beromi, G. W. Brudvig, N. Hazari, H. M. C. Lant, B. Q. Mercado, Angew. Chem. Int. Ed. 2019, $58,6094$.

[8] K. Osakada, T. Yamamoto, Coord. Chem. Rev. 2000, 198, 379.

[9] (a) M. Mohadjer Beromi, G. Banerjee, G. W. Brudvig, N. Hazari, B. Q. Mercado, ACS Catal. 2018, 8, 2526; (b)

D. J. Charboneau, G. W. Brudvig, N. Hazari, H. M. C. Lant, A. K. Saydjari, ACS Catal. 2019, 9, 3228.

[10] For representative examples, see: (a) R. L. Hartman, Org. Proc. Res. Devel. 2012, 16, 870; (b) K. F. Jensen, AlChE J. 2017, 63, 858; (c) D. Perera, J. W. Tucker, S. Brahmbhatt, C. J. Helal, A. Chong, W. Farrell, P. Richardson, N. W. Sach, Science 2018, 359, 429; (d) R. J. Perkins, A. J. Hughes, D. J. Weix, E. C. Hansen, Org. Proc. Res. Devel. 2019, 23, 1746.

[11] L. Huang, L. K. G. Ackerman, K. Kang, A. M. Parsons, D. J. Weix, J. Am. Chem. Soc. 2019, 141, 10978.

[12] Systems that utilize light, via a photocatalyst, or electricity have been reported. While these methods address some limitations associated with heterogeneous reductants, they have other practical challenges. For selected systems, see: (a) P. Zhang, C. C. Le, D. W. C. MacMillan, J. Am. Chem. Soc. 2016, 138, 8084; (b) R. J. Perkins, D. J. Pedro, E. C. Hansen, Org. Lett. 2017, 19, 3755; (c) H. Li, C. P. Breen, H. Seo, T. F. Jamison, Y.-Q. Fang, M. M. Bio, Org. Lett. 2018, 20, 1338; (d) T. J. DeLano, S. E. Reisman, ACS Catal. 2019, 9, 6751; (e) R. Martin-Montero, V. R. Yatham, H. Yin, J. Davies, R. Martin, Org. Lett. 2019, 21, 2947; (f) J. Yi, S. O. Badir, L. M. Kammer, M. Ribagorda, G. A. Molander, Org. Lett. 2019, 21, 3346; (g) Z. G. Brill, C. B. Ritts, U. F. Mansoor, N. Sciammetta, Org. Lett. 2020, 22, 410; (h) B. L. Truesdell, T. B. Hamby, C. S. Sevov, J. Am. Chem. Soc. 2020, 142, 5884.

[13] For select examples, see: (a) J. C. Tellis, D. N. Primer, G. A. Molander, Science 2014, 345, 433; (b) Z. Zuo, D. T. Ahneman, L. Chu, J. A. Terrett, A. G. Doyle, D. W. C. MacMillan, Science 2014, 345, 437; (c) S. A. Green, T. R. Huffman, R. O. McCourt, V. van der Puyl, R. A. Shenvi, J. Am. Chem. Soc. 2019, 141, 7709; (d) H. Yin, G. C. Fu, J. Am. Chem. Soc. 2019, 141, 15433.

[14] (a) K. Komeyama, R. Ohata, S. Kiguchi, I. Osaka, Chem. Commun. 2017, 53, 6401; (b) K. Komeyama, Y. Yamahata, I. Osaka, Org. Lett. 2018, 20, 4375; (c) K. Komeyama, T. Michiyuki, I. Osaka, ACS Catal. 2019, 9, 9285; (d) M. Ociepa, A. J. Wierzba, J. Turkowska, D. Gryko, J. Am. Chem. Soc. 2020, 142, 5355.

[15] (a) K. Takai, K. Nitta, O. Fujimura, K. Utimoto, J. Org. Chem. 1989, 54, 4732; (b) L. K. G. Ackerman, L. L. Anka-Lufford, M. Naodovic, D. J. Weix, Chem. Sci. 2015, 6, 1115; (c) J. L. Hofstra, A. H. Cherney, C. M. Ordner, S. E. Reisman, J. Am. Chem. Soc. 2018, 140, 139. 
[16] (a) M. Kuroboshi, M. Tanaka, S. Kishimoto, K. Goto, M. Mochizuki, H. Tanaka, Tetrahedron Lett. 2000, 41, 81; (b) A. García-Domínguez, Z. Li, C. Nevado, J. Am. Chem. Soc. 2017, 139, 6835; (c) W. Shu, A. García-Domínguez, M. T. Quirós, R. Mondal, D. J. Cárdenas, C. Nevado, J. Am. Chem. Soc. 2019, 141, 13812.

[17] In some cases, $N$-(3-bromopropyl)phthalimide was used as the alkyl electrophile because the polar functional group assists with isolation.

[18] An alternative method for optimizing reactions involves utilizing additional equivalents of the alkyl electrophile. See the SI for further details.

[19] H. Yin, J. Sheng, K.-F. Zhang, Z.-Q. Zhang, K.-J. Bian, X.-S. Wang, Chem. Commun. 2019, $55,7635$.

[20] S. D. Roughley, A. M. Jordan, J. Med. Chem. 2011, 54, 3451.

[21] F. Alonso, I. P. Beletskaya, M. Yus, Chem. Rev. 2002, 102, 4009.

[22] H.-Q. Do, E. R. R. Chandrashekar, G. C. Fu, J. Am. Chem. Soc. 2013, 135, 16288.

[23] (a) V. Bacauanu, S. Cardinal, M. Yamauchi, M. Kondo, D. F. Fernández, R. Remy, D. W. C. MacMillan, Angew. Chem. Int. Ed. 2018, 57, 12543; (b) C. Xu, W.-H. Guo, X. He, Y.-L. Guo, X.-Y. Zhang, X. Zhang, Nature Commun. 2018, 9, 1170.

[24] (a) F. Lovering, J. Bikker, C. Humblet, J. Med. Chem. 2009, 52, 6752; (b) F. Lovering, Med. Chem. Comm. 2013, 4,515 .

[25] P. S. Kutchukian, J. F. Dropinski, K. D. Dykstra, B. Li, D. A. DiRocco, E. C. Streckfuss, L.-C. Campeau, T. Cernak, P. Vachal, I. W. Davies, S. W. Krska, S. D. Dreher, Chem. Sci. 2016, 7, 2604.

[26] S. W. Krska, D. A. DiRocco, S. D. Dreher, M. Shevlin, Acc. Chem. Res. 2017, 50, 2976.

[27] (a) F. Reck, F. Zhou, C. J. Eyermann, G. Kern, D. Carcanague, G. Ioannidis, R. Illingworth, G. Poon, M. B. Gravestock, J. Med. Chem. 2007, 50, 4868; (b) T. Komine, A. Kojima, Y. Asahina, T. Saito, H. Takano, T. Shibue, Y. Fukuda, J. Med. Chem. 2008, 51, 6558.

[28] R. D. Taylor, M. MacCoss, A. D. G. Lawson, J. Med. Chem. 2014, 57, 5845.

[29] P. G. Jessop, Green Chem. 2011, 13, 1391.

[30] M. Mendel, I. Kalvet, D. Hupperich, G. Magnin, F. Schoenebeck, Angew. Chem. Int. Ed. $2020,59,2115$.

[31] I. Kalvet, G. Magnin, F. Schoenebeck, Angew. Chem. Int. Ed. 2017, 56, 1581.

[32] (a) L.-Y. He, M. Schulz-Senft, B. Thiedemann, J. Linshoeft, P. J. Gates, A. Staubitz, Eur. J. Org. Chem. 2015, 2015, 2498; (b) J. W. B. Fyfe, N. J. Fazakerley, A. J. B. Watson, Angew. Chem. Int. Ed. 2017, $56,1249$.

[33] K. Lin, R. J. Wiles, C. B. Kelly, G. H. M. Davies, G. A. Molander, ACS Catal. 2017, 7, 5129.

[34] (a) S.-i. Mho, B. Ortiz, N. Doddapaneni, S. M. Park, J. Electrochem. Soc. 1995, 142, 1047; (b) B. Eberle, O. Hübner, A. Ziesak, E. Kaifer, H.-J. Himmel, Chem. Eur. J. 2015, 21, 8578.

[35] J. Demarteau, A. Debuigne, C. Detrembleur, Chem. Rev. 2019, 119, 6906.

[36] F. T. T. Ng, G. L. Rempel, C. Mancuso, J. Halpern, Organometallics 1990, 9, 2762. 


\section{TOC Graphic}

Rationally Designed $C\left(s p^{2}\right)-C\left(s p^{3}\right)$ Cross-Electrophile Coupling...

Broad and expanded
substrate scope

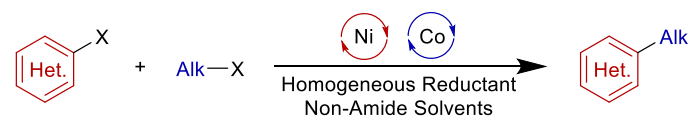
Non-Amide Solvents

$\begin{gathered}\text { One-pot dialkylation of } \\ \text { bromo(iodo)arenes }\end{gathered}$
$\begin{gathered}4 \text { examples } \\ (82 \% \text { avg yield) }\end{gathered}$

